14

\title{
TRANSKULTURELLE VERMITTLUNG VON LITERATUR - DIE KOMPETENZFÖRDERNDE ERFAHRUNG DES ÄSTHETISCHEN LERNENS
}

\author{
TRANSCULTURAL MEDIATION OF LITERATURE - \\ THE COMPETENCE ORIENTED EXPERIENCE OF AESTHETIC \\ LEARNING
}

\author{
Jelena Knežević \\ Universität Montenegro, Philosophische Fakultät, Nikšić, Montenegro \\ University of Montenegro, Faculty of Philology, Nikšić, Montenegro
}

\section{Zusammenfassung}

Im Rahmen dieses Aufsatzes werden einige wichtige Aspekte des ästhetischen Lernens bei der transkulturellen Vermittlung von Literatur dargestellt, mit dem Ziel zu zeigen, wie die systematische Interpretation ausgezeichneter literarischer Werke in der Ausbildung von Lehrenden zur humanistischen Bildung bzw. transkulturellen Vermittlung humanistischer Werte beiträgt, während gleichzeitig die Sprachkompetenzen der Lernenden gefördert werden. Die vorliegende Untersuchung fand im Rahmen eines universitären Unterrichtsprojektes statt. Sie zeigt auf, wie die ästhetische Erfahrung bei der literarischen Übersetzung durch den spielerischen Umgang mit Sprache ermöglicht wird sowie durch die Auseinandersetzung mit gelungenen sprachlich vermittelten Bildern, Sinnbildern und Metaphern, die nicht unbedingt kulturologisch geprägt sind, sondern vielmehr mit der Transformation von der persönlichen zur universalmenschlichen Erfahrung zu tun haben.

\section{Einführung}

Im Rahmen dieses Beitrags werden unterschiedliche Aspekte eines Unterrichtsprojektes analysiert, das an der Abteilung für deutsche Sprache und Literatur der Universität Montenegro realisiert wurde. Das Projekt zur deut-

\section{Abstract}

The paper deals with some important aspects of aesthetic learning within the transcultural teaching of literature aiming to show how the systematic interpretation of the best literary works as part of teacher education contributes to humanistic education or rather the transcultural mediation of humanistic values while at the same time language skills of learners being encouraged. The research was conducted as part of the university teaching project. It has shown how the playful use of language within the literary translation enables the aesthetic experience. Moreover, the evaluation confirmed that dealing with linguistically successful conveyed images, symbols and metaphors that are not necessarily culturally shaped, but rather related to conversion of personal to universal human experience fosters the transformation of aesthetic experience into cognition about the universal values beyond national borders.

schen Migrationsliteratur mit Jugoslawien-Bezug unter dem Titel Exil in der Sprache hatte in erster Linie zum Ziel, Studierende der Germanistik mit der Diversität der zeitgenössischen deutschen Literatur, in der interkulturelle Aspekte eine wesentliche Rolle spielen, vertraut zu machen. Weitere wichtige Lernziele waren 
die Entwicklung eines Verständnisses für die Bereicherung durch Internationalisierung und der Erwerb interkultureller Kompetenz und Übersetzungskompetenzen. Das Projekt verband Sprach- mit Literaturvermittlung und ermöglichte intensiven Spracherwerb auch im Literaturunterricht sowie den Erwerb praktischer Kompetenzen orientiert an den den aktuellen beruflichen Anforderungen entsprechenden erforderlichen Fähigkeiten. Diese Arbeit versteht sich auch als ein Versuch, den empirischen Einsatz interkultureller Literatur im Unterricht aus der Perspektive des ästhetischen Lernens zu reflektieren.

\section{Begriffsklärung}

Im Unterschied zum traditionellen Literaturunterricht, der sich am Einsatz literaturhistorischer Handbücher orientiert und den Text rein philologisch als selbstreferentielles Gebilde mit ästhetischem Anspruch versteht, fokussierten wir uns im Rahmen des Projektes auf das reflektierende Potenzial von Literatur, das sich im Akt des Lesens realisiert. Diesem rezeptionsästhetischen Literaturverständnis zufolge entsteht der ästhetische Gehalt erst durch die Kommunikation zwischen Text und Leser_in bzw. im Prozess des Lesens, der seinerseits von den gesellschaftlichen Bedingungen abhängig ist. Da Literatur individuelle und soziale Kommunikation anregen kann, befindet sie sich im Feld der gesellschaftlichen Repräsentation. Sie bildet Werte und Ideologien ab, gestaltet individuelle Denkweisen und nimmt an der Institutionalisierung von Kultur durch die Konstruktion des kollektiven Bewusstseins teil. /1/ Als „Form sprachlich-medialer Kommunikation und als Medium kultureller Deutungsmuster und bildungsrelevanter Inhalte, die gebraucht, aber auch reflektiert und weiterentwickelt werden können", so Altmayer, ist Literatur ein bedeutendes Instrument der ästhetischen Bildung. /2/ Ästhetische Bildung verstehen wir hier in weitestem Sinne als Konzept, das die Bedeutsamkeit der ästhetischen Erfahrung für die Weltbild- und Begriffsbildung sowie die Persönlichkeitsentwicklung betont. /3/ Anhand des Einbezugs von kognitiven, emotionalen und imaginativen Elementen übermittelt sinnliche Wahrnehmung beim Lesen multiple Möglichkeiten ISSN 1330-0067 der Sinnstiftung. Die Sinnstiftung umfasst ethische, soziale, religiöse und interkulturelle Aspekte, fordert die Bewertungsfähigkeit heraus und fördert die ganzheitliche Bildung persönlicher Identität. /4/ Die Rezeptionsästhetik hat bereits gezeigt, wie dies in der Literatur ermöglicht wird: In einer Art „Wechselspiel zwischen subjektiver Imagination und genauer Wahrnehmung" /5/ gewinnen die Lesenden neue Einsichten und machen neue, ästhetische Erfahrungen, während sie sich emotional involvieren, in die Figuren hineinversetzen und bangen, hoffen, leiden oder sich mit den Heldinnen und Helden freuen /6/. /7/ Die ästhetische Erfahrung erschließt „neue, bisher nicht gekannte Möglichkeiten des Lebens" und ermöglicht die „Vorstellungen von dem, was anders und wie es anders sein könnte" /8/. Insofern kann sie auch als Alteritätserfahrung verstanden werden und als solche für die Identitätsbildung besonders bedeutend sein. Davon ausgehend wäre die Auseinandersetzung mit interkultureller bzw. transkultureller Literatur im Unterricht zur interkulturellen Vermittlung, Fremdverstehen und Gewinn interkultureller Kompetenzen in doppelter Hinsicht relevant, weil sie, so wie das ästhetische Lernen auch, das Aufeinandertreffen mit dem Anderen, Fremden sowie mittelbar auch mit sich selbst ermöglicht und somit auch identitätsprägend sein kann.

\section{Projektbeschreibung und Analyse}

Auf diesen Grundlagen erarbeiteten wir an der Abteilung für deutsche Sprache und Literatur der Philologischen Fakultät der Universität Montenegro ein Projekt zur neueren deutschen Literatur, in dessen Rahmen die Germanistikstudierenden mit Prosaauszügen junger deutscher Autorinnen und Autoren jugoslawischer Herkunft konfrontiert wurden. Das Unterrichtsprojekt mit dem Titel „Exil in der Sprache" verband Sprach- mit Literaturunterricht und hat sich mehrere Lernziele eingesetzt, neben anderen vor allem mit der Diversität der transkulturellen deutschen Literatur vertraut $\mathrm{zu}$ werden und damit die Enwicklung eines Verständnisses für die Bereicherung durch Internationalisierung bzw. Erwerb der interkulturellen Kompetenzen. Weitere Lernziele waren 
16

Spracherwerb und Erwerb von Übersetzungskompetenzen sowie praktischen Kompetenzen wie Konzipieren, Präsentieren, Reflektieren, Diskutieren, Arbeit mit einer speziellen Software für Untertitel.

Ausgehend von der Prämisse, dass man das Unbekannte am leichtesten durch das Bekannte kennenlernt und akzeptiert, wurden zunächst Auszüge aus den Romanen von Saša Stanišić, Marica Bodrožić, Melinda Nadj Abonji und Martin Kordić ausgewählt /9/, die sich auf den Studierenden bekannte Situationen, Personen oder Phänomene der jüngeren Balkangeschichte beziehen (z.B. Titos Tod, die Sängerin Lepa Brena, den Fluss Drina, Konflikte im Balkankrieg, die Brücke von Mostar). Es wurde darauf Bedacht genommen, dass die ausgewählte Auszüge aus Romanen auffällige interkulturelle Elemente bzw. Elemente beider Kulturkontexte - des deutschen und des serbischen/kroatischen oder bosnischen - beinhalten. /10/

Die Textausschnitte wurden an die Studierenden des dritten. Studienjahres (Deutschkenntnisse auf der Niveaustufe B.1. - B.2. ) verteilt und sie hatten zuerst eine Woche Zeit, sich mit den Texten selbstständig zu befassen. Im Rahmen eines Übersetzungsseminars wurden dann in insgesamt acht Unterrichtseinheiten die deutschen Ausgangstexte von Stanišić, Bodrožić und Nadj Abonji in Gruppenarbeit (fünf Studierende pro Gruppe) mit Unterstützung der Lehrperson durch die Studierenden ins Montenegrinische übersetzt. Diese Aufgabenstellung bietete die Möglichkeit, intensive Text- und Wortschatzarbeit zu leisten und grammatikalische Strukturen einzuüben. Der Übersetzungsprozess förderte die aktive Rezeption der Texte und regte die Kreativität und Einbildungskraft bei den Adressat_innen an. Nach jeder Unterrichtseinheit erhielten die Studierenden von der Lehrperson Instruktionen für weitere selbstständige Übersetzungsarbeit an den Texten. Den Studierenden wurde ganz bewusst nicht gesagt, dass es einige dieser Romane bereits in serbischer bzw. kroatischer Übersetzung gibt, um größere Freiheit für die Arbeit an den eigenen Texten zu gewährleisten. Übersetzung als Lehrmethode wurde nicht nur aus dem Grund gewählt, weil ihr eine sehr in- tensive, reflexive und kreative Auseinandersetzung mit Literatur immanent ist, während gleichzeitig neue Vokabel gelernt und die Fertigkeit Lesen geschult wird /11/, sondern auch als Paradigma des kulturellen Kontakts, einer hybriden kulturellen Form, wobei sowohl inhaltliche als formal-strukturelle Elemente und Werte aus mindestens zwei Kulturkontexten stammen. Insofern kann die Übersetzung für die Arbeit mit trans- bzw. interkultureller Literatur in mehrfacher Weise sehr sinnvoll eingesetzt werden. Der bekannte Kontext ermöglichte es den Studierenden nicht nur, die zu übersetzenden deutschen Ausgangstexte sprachlich leichter zu verstehen, sondern gleich das Gefühl zu entwickeln, es mit Eigenem zu tun zu haben, sodass das Fremde in den Texten (deutsche Sprache, Kulturkontext, usw.) auch früh zu Vertrautem wurde. Aus der Perspektive des ästhetischen Lernens betrachtet, ermöglichte die literarische Übersetzung einen spielerischen Umgang mit dem Fiktiven, eine Mitgestaltung des Werkes in eigener Sprache und damit die bessere Darstellbarkeit und ein Verständnis der wirklichen Welten, die dahinter stecken.

Neben den Texten von Stanišić, Bodrožić und Nadj Abonji wurde den Studierenden auch Videomaterial von Lesungen von Texten der Autoren Martin Kordić und Nicol Ljubić zur Verfügung gestellt. Durch das Anhören der gelesenen Texte wurde die Hörfertigkeit geschult. Im Rahmen des regulären Sprachunterrichts sahen die Studierenden in insgesamt vier Unterrichtseinheiten diese Video-Lesungen von Kordić und Ljubić und übersetzten die vorgelesenen Texte mit Unterstützung durch die Lehrperson. Die Studierenden hatten die Aufgabe, mithilfe einer Free Software für Untertitelung (subtitle edit) das Videomaterial mit Untertiteln zu versehen. Neben der Schulung der Fertigkeit Hören ermöglichten die gehörten Videoausschnitte eine noch intensivere literarische Kommunikation zwischen Texten und Adressatinnen und Adressaten durch das Gefühl, dass die Geschichte gerade ihnen erzählt wurde und dass sie direkt gefordert sind, den fiktiven Raum inhaltlich, mit bekannten und unbekannten Details, zu füllen. Die Aufgabenstellung der Untertitelung wurde hier als praktisches Knowhow und intermediale Kompetenz betrachtet, 
die es im didaktischen Lichte den Studierenden ermöglichte, die Beziehung zwischen Text und visuellen Medien zu verstehen.

Zusätzlich erhielten die Studierenden im Rahmen des regulären Literaturunterrichts (zwei Unterrichtseinheiten) eine Einführung in die interkulturelle deutssprachige Literatur. Der Begriff Transkulturalität wurde nach Wolfgang Welsch im Zusammenhang mit den „kulturübergreifenden und die kulturellen Grenzen durchdringenden Phänomene[n] der Gegenwartsmoderne" erklärt /12/ und interkulturelle bzw. transkulturelle Literatur im weitesten Sinne als Literatur bestimmt, die von Autor_innen verfasst wird, die mehreren Sprach- und Kulturkontexten angehören und diese in ihren Texten sinnvoll verbinden. Die Studierenden bekamen einen Input zu Migrantionsliteratur und wurden mit den unterschiedlichen Definitionen von "Gastarbeiterliteratur" bis "Chamisso-Literatur" vertraut gemacht. In Form einer Vorlesung wurde ihnen auch das Schaffen der fünf deutschen Autor_innen jugoslawischer Herkunft präsentiert, deren Texte sie übersetzten - Grundlagen ihrer Poetiken und jugoslawischer Motive sowie die dazugehörigen Kontexte in ihren Werken. Aufgrund der schon übersetzten Texte wurde eine lebhafte Diskussion zu den einzelnen Aspekten der Romane von Stanišić Wie der Soldat das Grammofon repariert, Nadj Abonji Tauben fliegen auf, Ljubić Als wäre es Liebe, Kordić Wie ich mir das Glück vorstelle und die Erzählung von Borožić Tito ist tot geführt. Diese lebhafte Diskussion konkretisierte einerseits sowohl inhaltliche als auch formale Besonderheiten der Texte und zeigte anderseits, dass es den Studierenden schwerfiel, die Autor_innen von ihren Protagonist_innen zu trennen, auch wenn die Lehrpersonen auf die ästhetische Autonomie der Texte hinwiesen und die Notwendigkeit einer gewissen Distanz für eine ästhetisch-kritische Auseinandersetzung mit den fremden und eigenen Handlungsund Lebensmustern durch Literatur betonten. Der Realitätsbezug in den Texten trug dazu bei, dass die meisten Studierenden eine emotionale Beziehung zu den dargestellten Kriegs- und Migrationserfahrungen entwickelten, insbesondere zu der Position der Protagonist_innen, die zwischen unterschiedlichen kulturellen Kontexten ihre neue Identität anhand einer fremden Sprache gestalteten. Die Studierenden verstanden, dass für alle diese Autor_innen gerade die deutsche Sprache das wichtigste Mittel geworden ist, in der sie die unbekannte Welt ihrer Migration verstehen konnten, während die Landschaft ihrer Herkunft die Optik geblieben ist, durch die sie diese neue Welt betrachteten. Die neue Sprache wurde mehr und mehr zu einem Mittel des Fabulierens, einem Mittel der Reflexion, Neugestaltung und Überwindung der gesamten verwirrenden Lebenserfahrungen und des Gefühls der Nicht-Zugehörigkeit. Die Sprache verband für die Autor_innen das Bekannte und Unbekannte miteinander, schuf Beziehungen und Verständnis und stiftete neuen Sinn. Das ist die Erfahrung, die auch die Studierenden in ihrer kreativen Arbeit mit den Übersetzungen machten: Die jugoslawischen Themen in der deutschen Sprache brachten sie in einer Art ästhetischen, produktiven Spiels zu den transkulturellen Denkmustern, besonders bei der Arbeit mit den Texten von Bodrožić und Stanišić.

Die Auseinandersetzung mit dem Roman von Nikol Ljubić betonte den Unterschied zwischen der Migrationsliteratur als Literatur, die eine authentische Migrationserfahrung mitteilbar macht, und der inter- oder transkulturellen Literatur, in der unabhängig vom Thema Migration transkulturelle Elemente prägend sind. Diese Elemente weisen immer auf die Gefährlichkeit eindimensionaler Identitäten hin, die auf nationale, religiöse oder einzelne gesellschaftliche Aspekte reduziert sind. Dies diente als Übergang zu einer Diskussion über den literarischen Wert von Migrationsliteratur, die in keiner Weise in Verbindung mit dem Thema Migration steht, sondern vor allem mit der Art und Weise ihrer Darstellung sowie indirekt mit ihrer Funktion, Kultur zu vermitteln. Die Studierenden wurden in diesem Zusammenhang mit dem Adelbert-von-Chamisso-Preis bekannt gemacht, mit dem die Robert Bosch Stiftung von 1985 bis 2017 auf Deutsch schreibende Autorinnen und Autoren nicht-deutscher Muttersprache auszeichnete. Die prämierten Autorinnen und Autoren mit ihren völlig unterschiedlichen 
18

kulturellen Hintergründen verbindet der meisterhafte Gebrauch der deutschen Sprache - der Sprache, in die sie eingewandert sind - bei der literarischen Gestaltung der erlebten oder erdachten Erfahrungen, die die Leserinnen und Leser zur Reflexion ihrer eigenen globalisierten interkulturellen Wirklichkeiten und universaler Fragestellungen des menschlichen Lebens anregt. Insofern sind ihre Werke nicht nur ein selbstverständlicher Bestandteil der deutschsprachigen Gegenwartsliteratur, sondern sie zählen auch zu der besten Literatur der Welt, die keine Ideologie, keinen politisch-moralischen Anspruch, keine geschichtliche Kontinuität und keine nationale Identität aufweisen muss.

In einer darauf folgenden ganztägigen Übersetzungswerkstatt unter der Leitung aller drei Lehrenden präsentierten die Studierenden ihre finalen Versionen der Übersetzungen, diese wurden im Plenum reflektiert, diskutiert, mit den bestehenden Übersetzungen /13/ verglichen und überarbeitet. Die gemeinsame redaktionelle Arbeit an den einzelnen sprachlichen Konstruktionen ermöglichte ein vertiefendes Textverstehen und einen reflektierenden Einblick in die Prozesse der Bedeutungsbildung und Vernetzung zu Sinnkomplexen, die sich individuell und kulturell vollziehen /14/. Die Studierenden merkten, dass die Texte auch auf sprachlicher Ebene interkulturell geprägt sind und gaben sich Mühe, dass die Übersetzungen dies auch widerspiegeln. Der Vergleich mit den bestehenden Übersetzungen diente als eine Art Selbstkorrektur, Studierende auf eigene Fehler aufmerksam zu machen, womit eine weitere Ebene der Reflexion ermöglicht wurde.

Die endgültige Version der übersetzten Texte wurde für eine öffentliche Lesung vorbereitet und Ausschnitte für eine zweisprachige Broschüre ausgewählt. Die Auswahl zeigte, dass die Studierenden sehr gut die (Schnitt-)Stellen der kulturellen Begegnungen in den Texten erkennen können sowie die Stellen, die für das Entstehen der ästhetischen Erfahrungen besonderes relevant sind bzw. das "Hinterfragen eigener Urteile und Einstellungen" provozieren /15/ und zur Selbstverständigung beitragen können. Gleichzeitig wurde außerdem ein Konzept für einen Literaturabend zur Chamisso-Li- teratur erarbeitet, der als abschließende Veranstaltung des Projektes geplant wurde. In den Tagen bis zur Veranstaltung übten die Studierenden individuell das Lesen der deutschen Texte mit einem deutschsprachigen Lektor bzw. einer deutschsprachigen Lektorin. Dadurch wurde die Lesekompetenz intensiv gefördert sowie die Aussprache geübt.

Der Literaturabend zum Thema "Exil in der Sprache. Deutsche Migrationsliteratur mit Jugoslawien-Bezug" fand im städtischen Kulturzentrum in Podgorica vor ca. 50 Gästen statt und war zweisprachig angelegt. Er war als Collage aus Vortrag, kommentierten Lesungen und einer Präsentation der Videomaterialien konzipiert. Die Germanistikstudierenden lasen die Texte der auf Deutsch schreibenden Autorinnen und Autoren jugoslawischer Herkunft im Original und in ihrer eigenen Übersetzung. Am Anfang und am Ende der Veranstaltung wurden zwei Video-Lesungen auf Deutsch mit montenegrinischer Untertitelung der Studierenden gezeigt. An die Gäste wurde die im Zuge des Projekts erstellte zweisprachige Broschüre verteilt. Die zweisprachige Lesung ermöglichte es, dass die deutsche Sprache als Sprache der Kultur und Literatur eine breitere Öffentlichkeit in Podgorica erreichte. Deutschsprachige Literatur wurde der Öffentlichkeit in Podgorica bei dieser Veranstaltung mit der Perspektive ihres inter- und transkulturellen Charakters vorgestellt und so wurden auch ein erhöhtes Interesses für die gegenwärtige deutschsprachige Literatur und eine indirekte Werbung für das Deutschlernen und GermanistikStudium geschaffen. Aus pädagogisch-didaktischer Sicht wurde durch die öffentliche Lesung erzielt, dass die Studierenden ihre Angst vor öffentlichen Auftritten und die Redeangst abbauten. Als "performatives Ereignis" wirkte sie „selbstreferentiell und wirklichkeitskonstituierend" /16/ sowohl auf die Studierenden als auch auf das Publikum. Das hat sich im Nachhinein während einer Evaluierungsrunde mit den teilnehmenden Studierenden erwiesen. Alle Studierende sagten, dass sie die zeitgenössische deutschsprachige Literatur mit den transkulturellen Elementen vor dem Projekt nicht gekannt hatten und auch überhaupt nicht gewusst hatten, dass Saša Stanišić und Martin Kordić zu deutschen und nicht $\mathrm{zu}$ bosnischen Autoren 
zählen. Die meisten Studierenden waren der Meinung, dass ihnen die bekannten Inhalte der Texte mit ihrem Jugoslawien-Bezug durch das weniger bekannte oder ganz unbekannte deutsche Vokabular und die Satzstrukturen, für die sie entsprechende Äquivalenz in ihrer eigenen Sprache finden mussten, die Möglichkeit bot, einen emotionalen Bezug zu ihren eigenen, durch Transkulturalität markierten Realität zu erstellen. Konfrontiert mit bekannten Lebensmustern der Protagonist_innen, mit ihren Konflikten, die sie in der neuen kulturellen und sprachlichen Umgebung erleben, konnten die Studierenden ihren Angaben nach zu urteilen das Erzählte emotional reflektieren, wurden jedoch auch über den aktiven Rezeptionsprozess (das Übersetzen) zu Bewertungen angeregt, weil sie nicht handelnd in ein Geschehen verstrickt wurden und damit auch eine gewisse Distanz bewahren konnten. /17/ Die Texte aller Autor_nnen, besonderes von Stanišić und Nadj Abonji, verstanden die Studierenden als einen kulturellen Begegnungsort. Durch ihre Auseinandetzung mit den Texten wurde ihnen ermöglicht, an diesem Raum teilzuhaben; das spielerische Handeln mit fiktiven Texten machte für sie die wirklichen Welten erklärbar oder gestaltbar. /18/

\section{Fazit}

Die Evaluierung, die im Rahmen einer protokollierten Diskussion stattfand, bot auch die Grundlage für die weiteren Überlegungen zu den didaktischen Wirkungen des Projektes und zu den folgenden Schlussfolgerungen. Das beschriebene Projekt verband erfolgreich sprachliches, interkulturelles und ästhetisch-literarisches Lernen und begeisterte Studierende für Literatur. /19/ Die aktive Teilnahme an der Lesung und am Projekt beeinflusste ihre Lernmotivation insgesamt positiv. Die Studierenden verbesserten ihre Sprachkenntnisse, indem sie ihren Wortschatz erweitern und vertieften konnten, außerdem wurden alle vier Fertigkeiten gefördert. Die bekannten Motive und Kontexte in der Literatur der Autor_innen mit jugoslawischem Migrationshintergrund brachte den Adressat_innen das Erzählte näher. Das Übersetzen ermöglichte ihnen eine intensivere
Wahrnehmung, beflügelte ihre subjektive Imagination und ermöglichte eine Reihe neuer (ästhetischer) Erfahrungen - des Krieges, der Migration, der Begegnung mit dem Anderen - sowie ihre bewusste Reflexion sowohl durch den Input zur interkulturellen Literatur als auch während der weiteren redaktionellen Arbeit an den Übersetzungen bzw. der Vorbereitung und Durchführung der öffentlichen Präsentation. Die reflektierten ästhetischen Erfahrungen führten zu einem neuen Verständnis sowohl für das Fremde als auch für das Eigene und zur (Um-)Gestaltung der individuellen Denkweisen der Studierenden.

Während der Arbeit mit den Texten konnten sich die Studierenden mit der Position der Erzählerin/des Erzählers identifizieren, die/der das Geschehene teilweise aus der Perspektive eines Kindes schildert, das die "fremde“ deutsche Sprache nicht nur erlernt, sondern diese auch $\mathrm{zu}$ seiner eigenen und wichtigsten Ausdrucksform gemacht hat. Für die Studierenden, die täglich mit den Schwierigkeiten des Deutschlernens konfrontiert sind, wirkte das zusätzlich motivierend.

Die Analyse des Projektes hat auch gezeigt, dass die Studierenden durch die Arbeit mit literarischen Texten in einer Art ästhetischem Spiel neben dem Verständnis für Normen, Konventionen und Werte der Zielregion, deren Sprache sie lernen, auch sogenannte "Soft Skills“ erwerben konnten (wie Präsentieren, Diskutieren, Vergleichen, Konzipieren und das Arbeiten mit der Untertitelungs-Software als intermediale Kompetenz), ohne dass der Literaturunterricht auf seine traditionelle Position im GermanistikStudium verzichten muss und den literarischen Kanon der exzellenten linear-ästhetischen Gegenstände vernachlässigt.

Es hat sich auch gezeigt, dass die gelungenen sprachlich vermittelten Bilder, Sinnbilder und Metaphern, die die Adressat_innen am tiefsten berührt haben, nicht unbedingt kulturologisch geprägt sind, sondern vielmehr mit der Transformation der persönlichen in eine universalmenschlichen Erfahrung zu tun haben. Auf diese Art und Weise wirkt gute Literatur, die ohne Ideologien und nationale Vorzeichen für die Überwindung der Differenzen zwischen Kulturen sensibilisiert und lehrt, dass trotz aller 
20

Unterschiede Menschen überall Menschen sind.

\section{Notes}

/1/ Luhmann, N. (1997). Die Gesellschaft der Gesellschaft. Frankfurt a. M.: Suhrkamp. S. 494. Laut Systemtheorie sind ästhetische Texte in der Lage, differenzierte Komplexitätsbeschreibungen der subjektiven und gesellschaftlichen Wirklichkeit zu leisten und in vielen Kontexten anschlussfähig arbeiten zu können.

/2/ Altmayer, Claus (2007). Von der Landeskunde zur Kulturwissenschaft - Innovation oder Modetrend? In: Germanistische Mitteilungen 65, S. 8.

/3/ „Der Begriff Ästhetische Bildung hat seinen kulturphilosophischen Hintergrund in Friedrich Schillers Schrift Über die ästhetische Erziehung des Menschen (1795) und bezeichnet einen Ansatz in den Erziehungswissenschaften, bei dem der Mensch sich durch sinnliche Erfahrungen bildet und entwickelt. Es geht hier nicht allein um die Erfahrungen, die an künstlerischen Werken gemacht werden, sondern ästhetische Bildung zielt auf die Bildung der reflexiven Wahrnehmungsund Empfindungsfähigkeit in allen Lebensbereichen ab." Dohrn, A. (2014) Leseförderung mit literarischen Texten im DaZ-Unterricht. In: Bernstein, N.; Lerchner, Ch. (Hg.) Ästhetisches Lernen im DaF-/DaZ-Unterricht, Göttingen: Universitätsverlag, S. 135-136. Zugänglich auf: https://docplayer.org/40421418-Aesthetischeslernen-im-daf-daz-unterricht.html_[23.7.2020]

/4/ Vgl. Huber, F. (2008). Durch Lesen sich selbst verstehen. Zum Verhältnis von Literatur und Identitätsbildung. Bielefeld: transcript; Spinner, K. H. (2008). Perspektiven ästhetischer Bildung. Zwölf Thesen. In: Vorst, C.; Grosser, S.; Eckhardt, J.; Burrichter, R. (Hrsg.): Ästhetisches Lernen. Fachdidaktische Grundfragen und praxisorientierte Konzepte im interdisziplinären Kontext von Lehrerbildung und Schule. Frankfurt a.M. u.a.: Lang, S. 923.; Müller-Michaels, H. (2009). Grundkurs Lehramt Deutsch. Stuttgart: Klett.

/5/ Spinner, K. H. (2008). ibidem, S. 14.

/6/ Schier, C. (2014). Ästhetische Bildung in DaF und im fremdsprachlichen Literaturunterricht als Grundlage für eine nachhaltige Allianz zwischen Denken und Empfinden. In: Bernstein, N.; Lerchner, Ch. (Hg.), ibidem, S. 7.

/7/ „Das Spielerische ist nicht nur eine (etwa Schiller verpflichtete) idealistische Bestimmung des Menschen, sondern könnte zunehmend eine Strategie darstellen, individuelle und soziale Anforderungen zu bewältigen - Räume der ästhetischen Erfahrung sind auch Denkräume." Oerter, R. (2002)
Kindheit. In: ders.; Montada, L. (Hg.), Entwicklungspsychologie. Weinheim: Beltz, S. 234.

18/ Kirchner, C.; Schiefer Ferarri, M.; Spinner, K. H. (Hg.) (2006). Ästhetische Bildung und Identität. Fächerverbindende Vorschläge für die Sekundarstufe I und II. München: kopaed.: S. 15.

19/ Stanišić, S. (2006). Wie der Soldat das Grammofon repariert. Roman. München: Luchterhand; Bodrožić, M. (2002). Tito ist tot. Erzählungen. Frankfurt am Main: Suhrkamp; Nadj Abonji, M. (2010). Tauben fliegen auf. Roman. Salzburg: Jung und Jung; Kordić, M. (2014). Wie ich mir das Glück vorstelle. Roman. München: Hanser; Ljubić, N. (2012). Als wäre es Liebe. Roman. Hamburg: Hoffmann und Campe.

/10/ Ein Beispiel dafür ist der Ausschnitt aus dem Roman von Stanišić, in dem der Protagonist, der junge Alexander, der wegen des Krieges in Bosnien mit seinen Eltern nach Deutschland flüchten musste, erzählt, dass er in der Schule zum Thema „Essen, ich habe dich gern“ anstatt über die deutsche Stadt, die er nicht gerne hatte, über Börek schrieb, ein bosnisches Essen („,über das Hackfleisch und den Yufka-Teig“), das er vermisst. Stanišić, S. (2006). Ibid, S. 139.

/11/ Das „Übersetzen ist die intensivste Weise, die ich kenne, einen Text zu lesen“ Draesner, Ulrike, 2008, zitiert nach: Pleß, U. Übersetzen von Liedern im DaF-Unterricht. In: Bernstein, N.; Lerchner, Ch. (Hg.), ibid, S. 167.

112/ Welsch, W. (1996). Grenzgänge der Ästhetik, Stuttgart: Reclam, S. 271.

/13/ Stanišić, S. (2014). Kako vojnik popravlja gramofon. Übersetzt von Kostić-Tomović, J. Beograd: Samizdat B92; Nađ Abonji, M. (2010). Golubije srce. Übersetzt von Dedović, D. Beograd: Laguna; Bodrožić, M. (2004). Tito je mrtav. Übersetzt von Bilopavlović Vuković, L.

/14/ Köhnen, R. (Hg.) (2011). Einführung in die Deutschdidaktik, Stuttgart-Weimar: Metzler, S. 138.

/15/ Schier, C. (2014). Ibid, S. 7.

/16/ Stöver-Blahak, A. (2014) Ästhetische Kommunikation im DaF-Unterricht, In: Bernstein, N.; Lerchner, Ch. (Hg.), ibid, S. 130.

/17/ Bredella, L. (2007). Die welterzeugende und die welterschließende Kraft literarischer Texte: Gegen einen verengten Begriff von literarischer Kompetenz und Bildung. In: Bredella, Lothar; Hallet, Wolfgang (Hg.): Literaturunterricht, Kompetenzen und Bildung. Trier: WVT, S. 82. Zitiert nach: Schier, C. (2014). Ibid, S. 14.

/18/ Vgl. Rupp, G. (1987). Kulturelles Handeln mit Texten. Fallstudien aus dem Schulalltag. Paderborn; Müller-Michaels, H. (2009). Grundkurs Lehramt Deutsch. Stuttgart: Klett, S. 34-53; 
Kammler, C. (Hrsg.) (2006). Literarische Kompetenzen - Standards im Literaturunterricht, Seelze. Zitiert nach: Köhnen, R. Literaturdidaktik. In: drs. (Hg.) ibid, S. 138.

/19/ „Es gibt eine große Anzahl von Möglichkeiten, durch ein sprachintegratives Unterrichtsprinzip ästhetisches Lernen und ästhetische Bildung im DaZ-Unterricht anhand des Einsatzes literarischer Texte auf- und auszubauen" Dohrn, A. (2014) Leseförderung mit literarischen Texten im DaZ-Unterricht. Bernstein, N.; Lerchner, Ch. (Hg.), ibid, S. 149.

/20/ „Ästhetisches Lernen hat auch im DaZ-Unterricht einen Mehrwert und nicht im Gegensatz zur zunehmenden Kompetenzorientierung, sondern als ihre notwendige Ergänzung im Zeitalter von Globalisierung, zunehmender Mehrsprachigkeit,
Interkulturalität und Informationsüberschwemmung zu sehen ist“. Dohrn, A. (2014). ibid, S. 149.

Literatur

1. Altmayer, C.; Dobstadt, M.; Riedner, R.; Schier, C. (Hg.) (2014): Neue Konzepte zur Rolle der Literatur in Deutsch als Fremdsprache und internationaler Germanistik. Tübingen: Stauffenburg Verlag.

2. Chiellino, C. (2000) Interkulturelle Literatur in Deutschland. Ein Handbuch. Stuttgart: Metzler

3. Honnef-Becker, I. (Hg.) (2007), Dialoge zwischen den Kulturen. Interkulturelle Literatur und ihre Didaktik. Baltmannsweiler: Schneider Verlag Hohengehren.

4. Spinner, Kaspar H: Kreativer Deutschunterricht. Identität - Imagination - Kognition. Seelze 2001. 